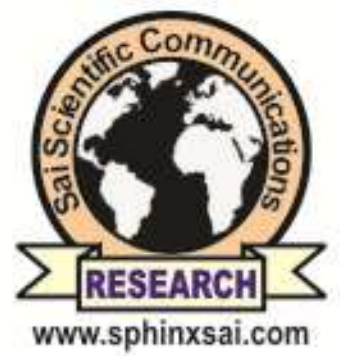

International Journal of PharmTech Research
CODEN (USA): IJPRIF, ISSN: 0974-4304, ISSN(Online): 2455-9563

\title{
Analysis of Carvedilol and Its Metabolite in Human Plasma Using Liquid Chromatography Coupled with Tandem Mass Spectrometry
}

\author{
Kalyan Chakravarthy Janjanam ${ }^{1}$ and \\ Bhavani Prasanna Kumar Bimireddy ${ }^{2}$
}

\author{
${ }^{1}$ University College of Pharmaceutical Sciences, Jawaharlal Nehru Technological \\ University, Kukatpally, Hyderabad-500 085, India. \\ ${ }^{2}$ Department of Pharmacy, Acharya Nagarjuna University, Nagarjuna Nagar, Guntur, \\ Andhra Pradesh -522510, India.
}

\begin{abstract}
A rapid, sensitive and selective method for the determination of Carvedilol and its metabolite in human plasma was developed using liquid chromatography with tandem mass spectrometry (HPLC-MS/MS). Carvedilol, its metabolite 4-OH carvedilol and abacavir (internal standard) were extracted from human plasma by solid phase extraction technique $(\mathrm{SPE})$ and analyzed on an Discovery column $(\mathrm{C} 8,50 \times 4.6 \mathrm{~mm}, 5 \mu)$ with the mobile phase of acetonitrile $-0.1 \%$ formic acid in water $(70: 30 \mathrm{v} / \mathrm{v})$. The analytes were detected using an electro spray ionization tandem mass spectrometry in the multiple reaction monitoring mode. The standard curve was linear $(\mathrm{r}=0.9998)$ over the concentration range of $0.5-100 \mathrm{ng} / \mathrm{mL}$ and $0.3-40 \mathrm{ng} / \mathrm{mL}$ for carvedilol and 4-OH carvedilol respectively. The lower limit of quantification for carvedilol was $0.5 \mathrm{ng} / \mathrm{mL}$ and $0.3 \mathrm{ng} / \mathrm{mL}$ for carvedilol and $4-\mathrm{OH}$ carvedilol respectively using $500 \mu \mathrm{L}$ plasma samples. The coefficient of variation and relative error for intra- and inter-assay at four QC levels were 3.37 to $9.53 \%, 4.76$ to $7.01 \%$ and 3.31 to $6.91 \%$, 5.23 to $6.64 \%$ respectively. The matrix effect for carvedilol, 4-OH carvedilol and abacavir were practically absent. The extraction recoveries of carvedilol, 4-OH carvedilol and abacavir were $78.90,83.25$ and $85.20 \%$, respectively.
\end{abstract}

Key wards : Carvedilol and its metabolite 4-OH carvedilol, Solid Phase Extraction (SPE), Method validation, LC-MS/MS.

\section{Introduction}

Carvedilol, 1-(9H-carbazol-4-yloxy)-3-[2-(2-methoxy-phenoxy)-ethyl amino]-propan-2-ol, is an antihypertensive agent with non-selective p-and 1 -adrenergic receptor blocking activities approved for the treatment of congestive heart failure. Carvedilol has been determined in biological fluids using highperformance liquid chromatography (HPLC) with fluorescence ${ }^{1-7}$, electrochemical detection ${ }^{8}$ or mass spectrometry (MS) ${ }^{9-11}$, capillary electrophoresis with UV detection ${ }^{12}$ and gas chromatography (GC) with 
MS detection ${ }^{13}$. Reverse- phase (RP)-HPLC methods were described for the analysis of Carvedilol racemate $^{1,3-5,8,9}$ or carvedilol enantiomers using chiral derivatization ${ }^{2,10}$. HPLC methods using a chiral stationary phase have been reported for the analysis of carvedilol enantiomers 6,7 . The clean-up procedures for the extraction of carvedilol from biological matrix consist of protein precipitation ${ }^{5,6,10,12}$, solid-phase extraction $(\mathrm{SPE})^{2,3}, 9$, liquid-liquid extraction $(\mathrm{LLE})^{6,7,11-13}$, combinations of protein precipitation with SPE ${ }^{4}$ or combinations of LLE with back-extraction ${ }^{1,8}$. Those methods use a large amount of biological samples $(0.15-$ $1.0 \mathrm{~mL}$ plasma or $2-5 \mathrm{~mL}$ urine samples) in order to obtain the high sensitivity or include time-consuming extraction procedures and/or relatively long run time. We adopted the simple and reliable approach for high throughput bioanalysis ${ }^{14-18}$ and presented a simple and sensitive method for simultaneous determination of carvedilol and its metabolite in human plasma. This method uses liquid chromatography (LC) combined with tandem mass spectrometry (MS). Samples are prepared using a solid phase extraction technique (SPE) since SPE has its advantageous which were discussed in earlier reports ${ }^{15-17}$. This method is useful for characterizing the pharmacokinetics of carvedilol and its metabolite in humans.

\section{Experimental}

\section{Chemicals and reagents}

Carvedilol (99.8\%), 4-OH carvedilol (99.5\%) and abacavir (99.2\%), Formic acid, ammonium formate were obtained from Sigma Aldrich, Bangalore, India. Water Oasis MCX cartridges were procured from Water, Bangalore, India. Acetonitrile, methanol and water were purchased from J.T Baker, Gurgaon, India. Drug-free human plasma containing $\mathrm{K}_{3}$ EDTA as the anticoagulant was obtained from Cauvery blood bank, Hyderabad, India.

\section{Preparation of calibration standards and quality control samples}

Primary stock solutions of carvedilol, $4-\mathrm{OH}$ carvedilol and abacavir $(1 \mathrm{mg} / \mathrm{ml})$ were prepared in methanol (diluent-1). Working standard solutions of carvedilol and 4-OH carvedilol were prepared by diluting each primary solution with $60 \%$ methanol in water (Diluent-2). The working solution for internal standard $(600 \mathrm{ng} / \mathrm{mL})$ was prepared by diluting an aliquot of stock solution with diluent -2 . All solutions were stored at $2-10{ }^{\circ} \mathrm{C}$ in polypropylene bottles in not in use. Human plasma calibration standards of carvedilol $(0.5$, $1.0,3.0,8.0,20.0,40.0,60,80$ and $100 \mathrm{ng} / \mathrm{mL})$ and $4-\mathrm{OH}$ carvedilol $(0.3,0.6,1.0,3.0,8.0,15.0,20.0,30.0$ and $40.0 \mathrm{ng} / \mathrm{mL}$ ) were prepared by spiking appropriate amount of the working standard solutions into a pool of 10 lots of drug-free human plasma. Quality control (QC) samples at $0.5,1.5,35.0,75.0 \mathrm{ng} / \mathrm{mL}$ for carvedilol and $0.3,0.9,13.0$, and $30.0 \mathrm{ng} / \mathrm{mL}$ were prepared in bulk by adding appropriate working standard solutions to drug free human plasma. The QC samples were aliquoted $(50 \mu \mathrm{l})$ into polypropylene tubes and stored at $-20 \circ \mathrm{C}$ until analysis.

\section{Sample preparation}

$200 \mu \mathrm{l}$ of blank plasma, calibration standards and QC samples were mixed with $25 \mu 1$ of internal standard working solution and $100 \mu \mathrm{l}$ of $0.1 \mathrm{~N}$ hydrochloric acid $(\mathrm{HCl})$ and vertex for proper mixing. The samples were extracted with ion exchange solid phase extraction technique which includes conditioning and equilibration of cartridge (Oasis, MCX, $30 \mathrm{mg}, 1 \mathrm{cc}$ ) with $2 \mathrm{~mL}$ of methanol and $2 \mathrm{~mL}$ of Milli-Q water followed by loading the prepared plasma sample. Washing step includes $1 \mathrm{~mL}$ of $0.1 \mathrm{~N} \mathrm{HCl}$ in water and $1 \mathrm{~mL}$ of Milli-Q water, elute with $2 \mathrm{~mL}$ of $2 \%$ ammonia in methanol and evaporated to dryness under nitrogen at $50^{\circ} \mathrm{C}$. The residues were dissolved in $250 \mu \mathrm{l}$ of reconstitution solution (mobile phase) by vortex for $40 \mathrm{~s}$, transferred to injection vials, and $10 \mu \mathrm{L}$ of samples were injected for LC-MS/MS analysis.

\section{LC-MS/MS analysis}

The chromatographic system used for LC-MS/MS analysis consisted of LC-10ADvp pumps, SIL-HTC auto sampler, CTO-10ASvp column oven and a SCL-10Avp system controller (Shimadzu). The separation was performed on Discovery column $(\mathrm{C} 8,50 \times 4.6 \mathrm{~mm}, 5 \mu)$ using a mixture of acetonitrile- $0.1 \%$ formic acid in water $(70: 30, \mathrm{v} / \mathrm{v})$ at a flow rate of $0.7 \mathrm{~mL} / \mathrm{min}$. The column and auto sampler tray were maintained at $40^{\circ} \mathrm{C}$ and $10^{\circ} \mathrm{C}$, respectively. The analytical run time was $2.0 \mathrm{~min}$. The eluent was introduced directly into the turbo ion spray source of a tandem quadrupole mass spectrometer (API 4000, Applied Biosystems/MDS SCIEX, USA). 
The turbo ion spray source was operated with typical settings as follows: ionization mode, positive; curtain gas, $15 \mathrm{psi}$; nebulizer gas, $40 \mathrm{psi}$; turbo gas, $55 \mathrm{psi}$; ion spray voltage, $5500 \mathrm{~V}$; temperature, $450{ }^{\circ} \mathrm{C}$. The molecular ions of carvedilol, 4-OH carvedilol and abacavir were formed using the declustering potentials of $80 \mathrm{~V}, 60 \mathrm{~V}$ and $60 \mathrm{~V}$ respectively, and their molecular ions were fragmented at collision energy of $35 \mathrm{~V}, 45 \mathrm{~V}$ and $25 \mathrm{~V}$ by collision-activated dissociation with nitrogen as the collision gas at a pressure setting of 6 on the instrument. Multiple reaction monitoring (MRM) mode was employed for the quantification: $\mathrm{m} / \mathrm{z} 407.2 \rightarrow 100.0$ for carvedilol, $423.3 \rightarrow 100.1$ for $4-\mathrm{OH}$ carvedilol and $\mathrm{m} / \mathrm{z} 287.20 \rightarrow 191.20$ for abacavir. Peak areas for all components were automatically integrated using Analyst software version 1.4.2 (Applied Biosystems/MDS SCIEX).

\section{Method validation}

The validation of the above method was carried out as per US FDA guidelines ${ }^{19}$. The parameters determined were selectivity, matrix effect, linearity, precision, accuracy, recovery, stability and dilution integrity.

\section{Results and discussions}

\section{Calibration curve performance}

This method is validated to meet the acceptance criteria of industrial guidance for the bioanalytical method validation. Calibration curves were obtained over the concentration range of 0.5 to $100 \mathrm{ng} / \mathrm{mL}$ and 0.3 to $40 \mathrm{ng} / \mathrm{mL}$ for carvedilol and 4-OH carvedilol, respectively in plasma (Table-1). Linear regression analysis with a weighting of $1 / \mathrm{x}^{2}$ gave the optimum accuracy of the corresponding calculated concentrations at each level. The low CV $(<15 \%)$ value for the slopes of carvedilol $(12.52 \%)$ and $4-\mathrm{OH}$ carvedilol $(12.55 \%)$ indicated the repeatability of the method.

Table-1: Calculated concentrations of carvedilol and 4-OH carvedilol in calibration standards $(\mathrm{n}=5)$

\begin{tabular}{|c|c|c|c|c|c|c|c|c|c|c|c|c|}
\hline \multicolumn{13}{|c|}{ Carvedilol } \\
\hline & CS-1 & CS-2 & CS-3 & CS-4 & CS-5 & CS-6 & CS-7 & CS-8 & CS-9 & \multirow{2}{*}{$\begin{array}{l}\text { Slope } \\
\text { (Mean) }\end{array}$} & \multirow{2}{*}{$\begin{array}{c}\text { Intercept } \\
\text { (Mean) }\end{array}$} & \multirow{2}{*}{$\begin{array}{c}\mathbf{R} \\
\text { (Mean) }\end{array}$} \\
\hline $\begin{array}{l}\text { Nominal } \\
(\mathrm{ng} / \mathbf{m L})\end{array}$ & 0.5 & 1.0 & 3.0 & 8.0 & 20.0 & 40.0 & 60.0 & 80.0 & 100.0 & & & \\
\hline Mean & 0.5 & 0.9 & 2.8 & 8.0 & 19.6 & 43.6 & 61.0 & 81.1 & 96.9 & \multirow{4}{*}{0.000191} & \multirow{4}{*}{-0.000493} & \multirow{4}{*}{0.9978} \\
\hline $\mathrm{SD}$ & 0.0 & 0.0 & 0.1 & 0.3 & 0.7 & 1.2 & 2.3 & 2.1 & 1.1 & & & \\
\hline$\% \mathrm{CV}$ & 1.8 & 4.5 & 4.5 & 4.2 & 3.7 & 2.7 & 3.8 & 2.6 & 1.1 & & & \\
\hline$\% \mathrm{NOM}$ & 104.5 & 93.4 & 94.8 & 100.2 & 98.1 & 108.9 & 101.7 & 101.4 & 96.9 & & & \\
\hline \multicolumn{13}{|c|}{ 4-OH Carvedilol } \\
\hline \begin{tabular}{|l} 
Nominal \\
$(\mathrm{ng} / \mathrm{mL})$
\end{tabular} & 0.3 & 0.6 & 1.0 & 3.0 & 8.0 & 15.0 & 20.0 & 30.0 & 40.0 & \multirow{5}{*}{0.00047} & \multirow{5}{*}{0.00411} & \multirow{5}{*}{0.9959} \\
\hline Mean & 0.3 & 0.7 & 1.1 & 3.2 & 8.3 & 14.5 & 17.9 & 27.7 & 40.8 & & & \\
\hline SD & 0.0 & 0.0 & 0.1 & 0.1 & 0.4 & 0.7 & 0.8 & 0.8 & 1.7 & & & \\
\hline$\% \mathrm{CV}$ & 1.3 & 2.9 & 4.9 & 3.6 & 4.6 & 4.7 & 4.4 & 2.8 & 4.0 & & & \\
\hline $\begin{array}{c}\% \\
\text { Nominal }\end{array}$ & 93.5 & 108.2 & 112.9 & 105.7 & 103.1 & 96.6 & 89.9 & 92.3 & 101.9 & & & \\
\hline
\end{tabular}


Table-2: Inter and intra-day precision and accuracy of carvedilol and 4-OH carvedilol

\begin{tabular}{|c|c|c|c|c|}
\hline \multicolumn{5}{|l|}{ Carvedilol } \\
\hline \multicolumn{5}{|c|}{ Inter-day precision and accuracy $(n=24)$} \\
\hline & LLOQ & LQC & MQC & HQC \\
\hline Nominal (ng/mL) & 0.5 & 1.5 & 34.5 & 74.0 \\
\hline Mean \pm sd & $0.5 \pm 0.04$ & $1.4 \pm 0.09$ & $37.9 \pm 1.25$ & $78.1 \pm 2.82$ \\
\hline$\% \mathrm{CV}$ & 6.9 & 6.9 & 3.3 & 3.6 \\
\hline$\%$ Nominal & 105.5 & 91.6 & 108.2 & 104.1 \\
\hline \multicolumn{5}{|c|}{ Intra-day precision and accuracy $(n=12)$} \\
\hline Mean \pm sd & $0.4 \pm 0.05$ & $1.3 \pm 0.09$ & $37.8 \pm 1.27$ & $78.1 \pm 3.20$ \\
\hline$\% \mathrm{CV}$ & 9.5 & 6.5 & 3.4 & 4.1 \\
\hline$\%$ Nominal & 102.0 & 90.8 & 109.5 & 105.5 \\
\hline \multicolumn{5}{|c|}{ 4-OH Carvedilol } \\
\hline \multicolumn{5}{|c|}{ Inter-day precision and accuracy $(n=24)$} \\
\hline & LLOQ & LQC & MQC & HQC \\
\hline Nominal (ng/mL) & 0.3 & 0.9 & 13.0 & 30.0 \\
\hline Mean \pm sd & $0.3 \pm 0.02$ & $0.9 \pm 0.06$ & $13.0 \pm 0.76$ & $30.3 \pm 1.71$ \\
\hline$\% \mathrm{CV}$ & 5.2 & 6.6 & 5.9 & 5.6 \\
\hline$\%$ Nominal & 102.2 & 100.4 & 100.1 & 101.0 \\
\hline \multicolumn{5}{|c|}{ Intra-day precision and accuracy $(n=12)$} \\
\hline Mean \pm sd & $0.3 \pm 0.01$ & $0.9 \pm 0.06$ & $13.2 \pm 0.9$ & $30.3 \pm 2.12$ \\
\hline$\% \mathrm{CV}$ & 4.7 & 6.9 & 6.8 & 7.0 \\
\hline$\%$ Nominal & 105.0 & 100.5 & 101.5 & 100.9 \\
\hline
\end{tabular}

\section{Accuracy and precision}

The inter day accuracy and precision results are for carvedilol and 4-OH carvedilol are presented in Table-2, the assay was both accurate and precise between runs and within individual runs for each level. The greatest mean inter day percent deviation was $6.91 \%$ (carvedilol) and 5.23\% (4-OH carvedilol) for LLOQ. All non LLOQ QC levels had inter- day percent deviations less than $6.85 \%$ and $6.64 \%$. The intra day precision for the non LLOQ QCs was less than $9.53 \%$ \& $4.71 \%$ and all other level of QCs had intra day percent deviation less than $6.49 \%$ \& $7.01 \%$ for carvedilol and 4-OH carvedilol, respectively.

\section{Stability in plasma}

The stability of carvedilol and 4-OH carvedilol in plasma matrix was also investigated as described above. Any deterioration of carvedilol and its metabolite during freeze-thaw cycles, extended time on counter top (bench top) was monitored. Four freeze thaw cycles (where the samples were completely thawed and refrozen for four times) and 9 hrs stability check at ambient temperature were performed. There was no deterioration in carvedilol and its metabolite at either QC level (low and high) for the various freeze thaw cycles, suggesting that drug concentrations can be confidently determined in samples that had been previously thawed and kept at ambient temperature was performed since the plasma sample could conceivably stand on bench for up to $9 \mathrm{hrs}$ after thawing or before freezing.

\section{Stability in extract}

The stability of carvedilol and its metabolite was also tested as described above. There was no significant deterioration (less than $2.83 \%$ for carvedilol and $1.31 \%$ for its metabolite) was observed in dry extract at both QC concentrations (low and high) during $24 \mathrm{hrs}$ storage at 2-8 ${ }^{0} \mathrm{C}$. Apart from the dry extract stability, auto sampler stability was also performed at $10^{\circ} \mathrm{C}$. The $\%$ change was observed less than $2.84 \%$ and $0.28 \%$ for carvedilol and its metabolite, respectively after $32 \mathrm{hrs}$ auto sampler storage. The extract stability at ambient temperature was not assessed. The stability data was represented in Table-3. 
Table-3: Stability data of carvedilol and 4-OH carvedilol

\begin{tabular}{|c|c|c|c|c|}
\hline Stability & Conc. $(\mathbf{n g} / \mathbf{m L}) \pm \mathbf{s d}$ & \% Stability & Conc. $(\mathbf{n g} / \mathbf{m L}) \pm \mathbf{s d}$ & \% Stability \\
\hline \multicolumn{5}{|c|}{ Carvedilol } \\
\hline BTS & $1.47 \pm 0.07$ & 97.7 & $77.16 \pm 3.49$ & 102.9 \\
\hline DES & $1.44 \pm 0.03$ & 96.1 & $79.4 \pm 4.14$ & 105.8 \\
\hline FTS & $1.44 \pm 0.06$ & 96.2 & $76.11 \pm 2.53$ & 101.5 \\
\hline IIS & $1.44 \pm 0.09$ & 95.9 & $79.39 \pm 1.59$ & 105.9 \\
\hline \multicolumn{5}{|c|}{ 4 OH Carvedilol } \\
\hline BTS & $0.94 \pm 0.04$ & 104.4 & $31.37 \pm 0.87$ & 104.6 \\
\hline DES & $0.92 \pm 0.05$ & 101.6 & $31.17 \pm 1.86$ & 103.9 \\
\hline FTS & $0.91 \pm 0.04$ & 101.2 & $31.38 \pm 0.34$ & 104.6 \\
\hline IIS & $0.92 \pm 0.03$ & 102.7 & $31.44 \pm 1.28$ & 104.8 \\
\hline
\end{tabular}

\section{Specificity}

During the validation, blank plasma samples from (8) different lots were evaluated and all plasma lots were found to be satisfactory. There were no interfering peaks in the blank plasma at the retention times of carvedilol and 4-OH carvedilol and internal standard. During the specificity run, an LLOQ standard was extracted and injected. The responses for the blank plasma from the eight (8) different lots were compared to the LLOQ standard of the two analytes and internal standard. No significant response $(\leq 20 \%$ for the analyte response and $\leq 5 \%$ of the internal standard response) was observed at the retention time of the analytes and internal standard in the blank plasma as compared to the LLOQ standard.

The method is validated for selectivity also, selectivity is the ability of the bio-analytical method to measure and differentiate the analytes in the presence of components that may be expected to be present. These could include metabolites, impurities, matrix components, or any other concomitantly administered drugs. In this experiment, Interference at one analyte retention time (RT) was checked by spiking of highest calibration standard of other analyte of interest and vice versa in six screened blank plasma. Similarly Interference at analyte RT was checked by spiking the working range of internal standard in six screened blank plasma. There was no significant interference was observed at analytes RT and internal standard R.T. The sample chromatogram of carvedilol and $4 \mathrm{OH}$ carvedilol were represented in figure $1 \& 2$. 
A
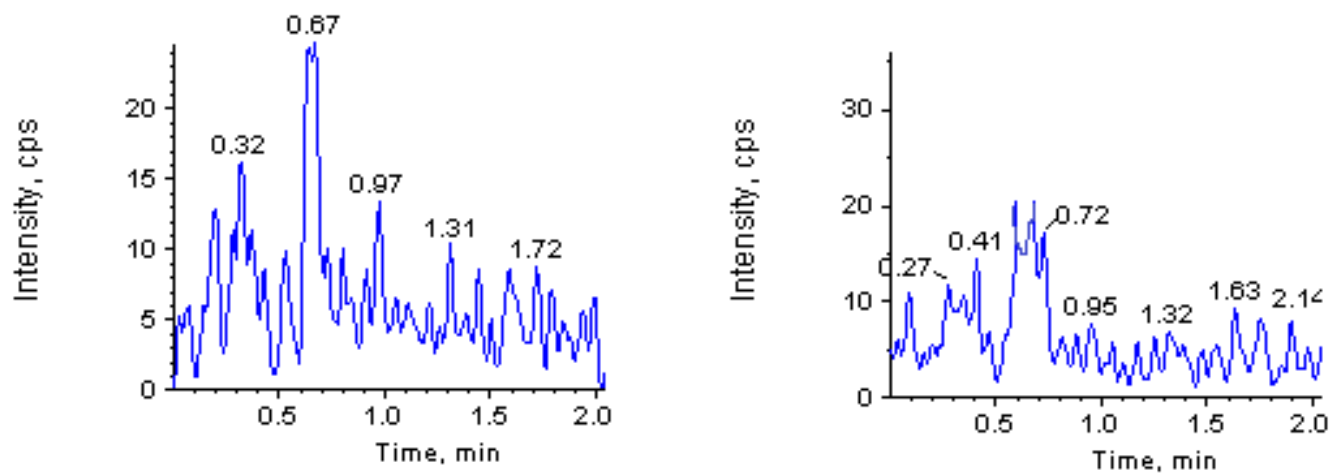

B
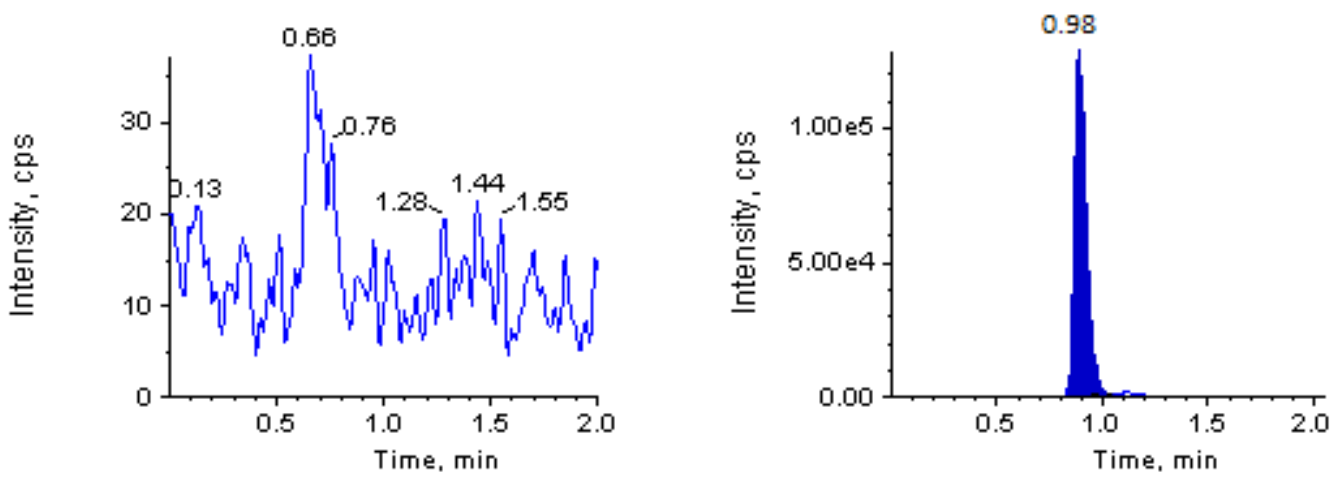

C
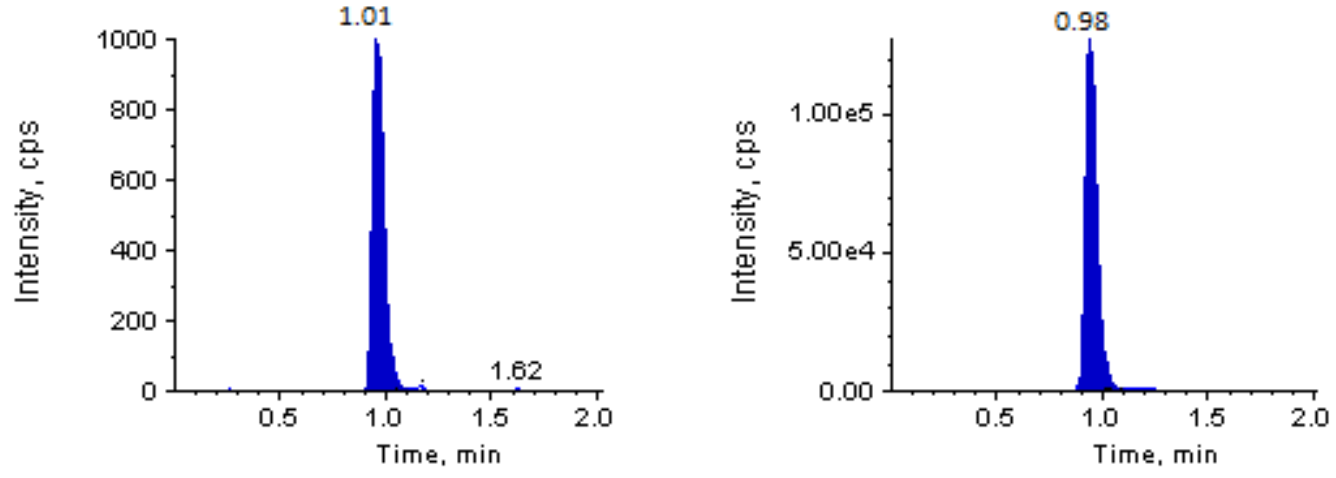

Figure-1: Chromatogram of Carvedilol, A) Double Blank, B) Plasma blank + IS, C) LLOQ 

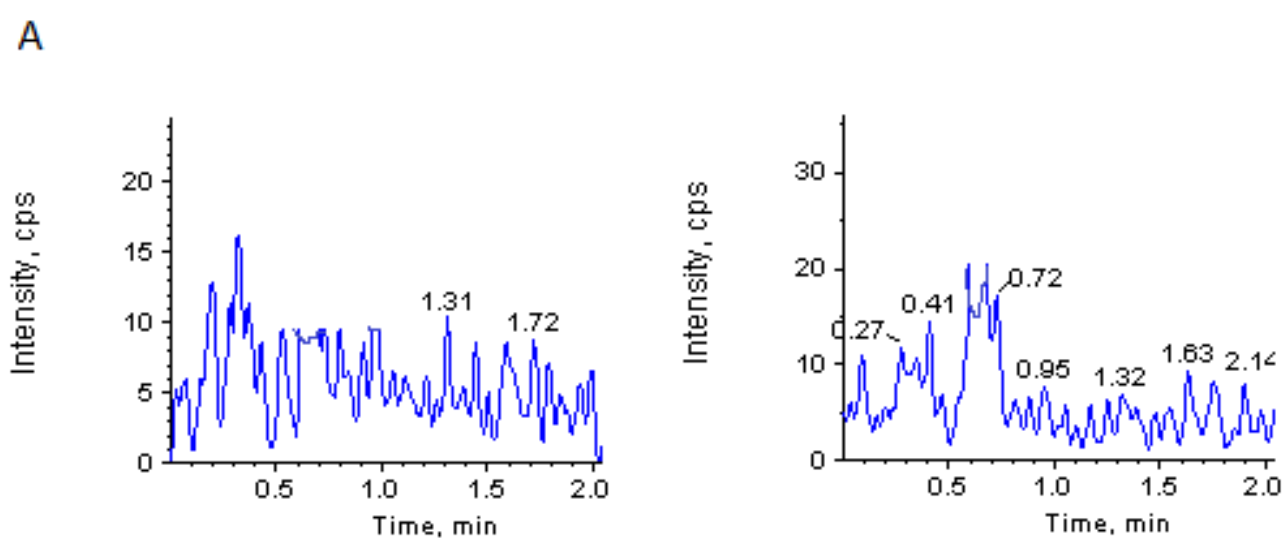

B
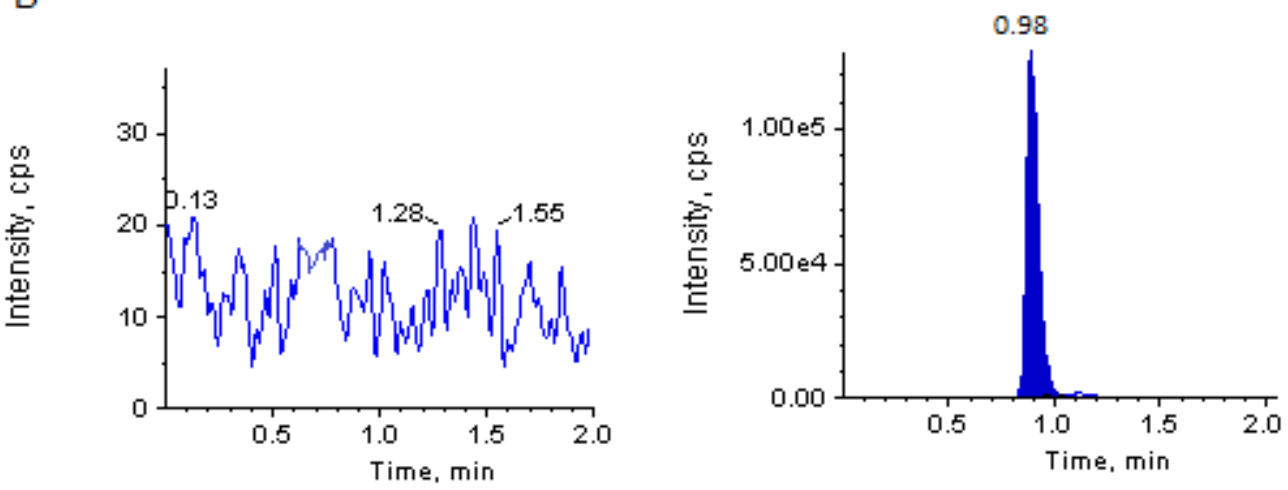

$\mathrm{C}$
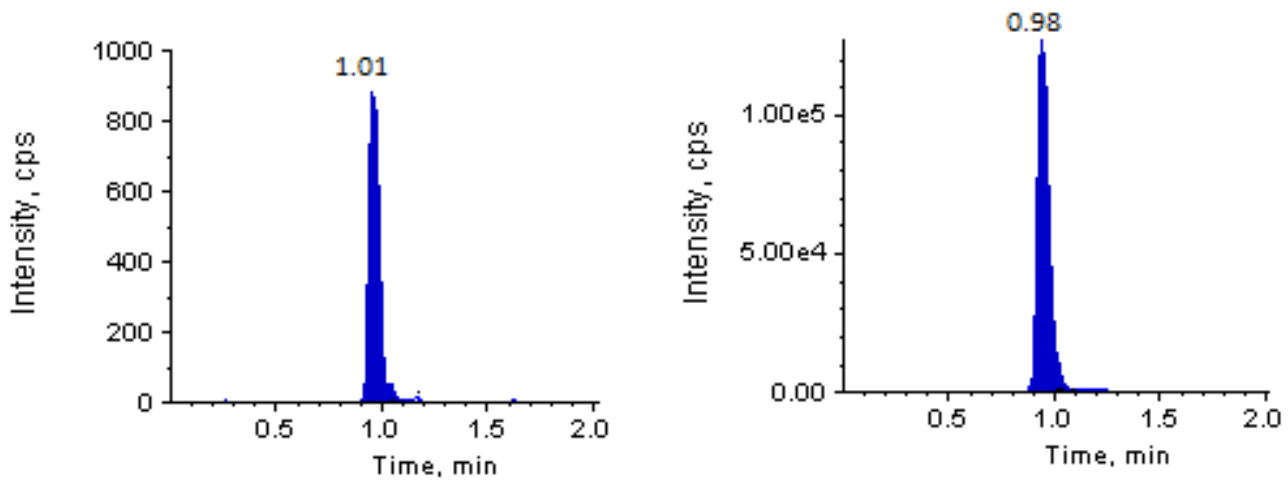

Figure-2: Chromatogram of 4-OH Carvedilol, A) Double Blank, B) Plasma blank + IS, C) LLOQ

\section{Matrix effect}

The direct or indirect alteration or interference in response due to the presence of unintended analytes (for analysis) or other interfering substances in the sample, resulting ion suppression or enhancement, which tends to give unexpected response. Matrix effect experiment was performed by taking six different lots of blank plasma from different source in duplicate and extracted according to the analytical method procedure. The analytes and internal standard were spiked into the extracted plasma blank to obtain the post extracted LQC, MQC and HQC samples. The post extracted QC samples were analyzed along with the aqueous samples equivalent to $\mathrm{LQC}, \mathrm{MQC}$ and $\mathrm{HQC}$ concentrations and compared the response of the post extracted to the un-extracted samples. There was no significant ion suppression or enhancement was observed at analytes RT and internal standard RT. The matrix factor was within acceptable limit (0.8 to 1.2) for both carvedilol and 4- $\mathrm{OH}$ carvedilol respectively. There was no significant ion enhancement or suppression was observed for 
internal standard. The recoveries of the carvedilol, 4-OH carvedilol and abacavir were 78.90, 83.25 and $85.20 \%$, respectively. The results were summarized in Table- 4 .

Table-4: Matrix effect data of carvedilol and 4-OH carvedilol

\begin{tabular}{|c|c|c|c|c|c|c|}
\hline \multirow{4}{*}{} & \multicolumn{3}{|c|}{ Carvedilol } & \multicolumn{3}{c|}{ 4-OH Carvedilol } \\
\cline { 2 - 3 } & \multicolumn{2}{|c|}{ Mean Response } & \multirow{2}{*}{ MF } & \multicolumn{2}{c|}{ Mean Response } & \multirow{2}{*}{ MF } \\
\cline { 2 - 3 } \cline { 5 - 6 } & $\begin{array}{c}\text { (Neat } \\
\text { Sample) }\end{array}$ & $\begin{array}{c}\text { (Post } \\
\text { Extracted) }\end{array}$ & & $\begin{array}{c}\text { (Neat } \\
\text { Sample) }\end{array}$ & $\begin{array}{c}\text { (Post } \\
\text { Extracted) }\end{array}$ & \\
\hline LLOQ QC & 5271 & 5632 & 1.07 & 17386 & 18720 & 1.08 \\
\hline LQC & 168431 & 175523 & 1.04 & 51610 & 54962 & 1.06 \\
\hline MQC & 3832059 & 3978402 & 1.04 & 652599 & 691232 & 1.06 \\
\hline HQC & 8221457 & 8422883 & 1.02 & 1535437 & 1312516 & 0.85 \\
\hline
\end{tabular}

\section{Conclusion}

A rapid, sensitive and reliable LC-MS/MS method for the determination of carvedilol and its metabolite in human plasma has been successfully developed and validated. This assay method demonstrated acceptable sensitivity, precision, accuracy, selectivity, recovery and stability and negligible matrix effect. The validated method can successfully apply to assay human plasma samples from the clinical study of carvedilol.

\section{References}

1. F. Varin, L.X. Cubeddu and J. Robert Powell, J. Pharm. Sci., 75, 1195 (1986); doi:10.1002/jps.2600751218.

2. E.J. Eisenberg, W.R. Patterson and G.C. Kahn, J. Chromatogr. B, 493, 105 (1989); doi:10.1016/S03784347(00)82713-9.

3. N. Hokama, N. Hobara, H. Kameya, S. Ohshiro and M. Sakanashi, J. Chromatogr. B Biomed. Sci. Appl, 732, 233 (1999); doi:10.1016/S0378-4347(99)00248-0.

4. F. Behn, S. Laer and H. Scholz, J. Chromatogr. Sci., 39, 121 (2001); doi:10.1093/chromsci/39.3.121.

5. P. Ptacek, J. Macek and J. Klima, J. Chromatogr. B Analyt. Technol. Biomed. Life Sci., 789, 405 (2003); doi:10.1016/S1570-0232(03)00078-3.

6. L. Clohs and K.M. McErlane, J. Pharm. Biomed. Anal., 31, 407 (2003); doi:10.1016/S07317085(02)00609-X.

7. M. Saito, J. Kawana, T. Ohno, M. Kaneko, K. Mihara, K. Hanada, R. Sugita, N. Okada, S. Oosato, M. Nagayama, T. Sumiyoshi and H. Ogata, J. Chromatogr. B Analyt. Technol. Biomed. Life Sci., 843, 73 (2006); doi:10.1016/j.jchromb.2006.05.018.

8. M. Machida, M. Watanabe, S. Takechi, S. Kakinoki and A. Nomura, J. Chromatogr. B Analyt. Technol. Biomed. Life Sci., 798, 187 (2003); doi:10.1016/j.jchromb.2003.09.039.

9. M. Gergov, J.N. Robson, E. Duchoslav and I. Ojanpera, J. Mass Spectrom., 35, 912 (2000); doi:10.1002/1096-9888(200007)35:7<912::AID-JMS19>3.0.CO;2-4.

10. E. Yang, S. Wang, J. Kratz and M.J. Cyronak, J. Pharm. Biomed. Anal., 36, 609 (2004); doi:10.1016/j.jpba.2004.07.008.

11. N.C. do Carmo Borges, G. Duarte Mendes, D. de Oliveira Silva, V. Marcondes Rezende, R.E. Barrientos-Astigarraga and G. De Nucci, J. Chromatogr. B Analyt. Technol. Biomed. Life Sci., 822, 253 (2005); doi:10.1016/j.jchromb.2005.06.012.

12. L. Clohs and K.M. McErlane, J. Pharm. Biomed. Anal., 24, 545 (2001); doi:10.1016/S07317085(00)00468-4. 
13. S.W. Myung and C.H. Jo, J. Chromatogr. B Analyt. Technol. Biomed. Life Sci., 822, 70 (2005); doi:10.1016/j.jchromb.2005.05.023.

14. Y. Zhang, Y. Han, S.P. Putluru, M.K. Matta, P. Kole, S. Mandlekar, M. Furlong, T. Liu, R. Iyer, P. Marathe, Z. Yang, Y. Lai and D. Rodrigues, Drug Metab. Dispos., 44, 320 (2016); doi:10.1124/dmd.115.066944.

15. M.K. Matta, L. Burugula, N.R. Pilli, J.K. Inamadugu and S.R. Jvln, Biomed. Chromatogr., 26, 1202 (2012); doi:10.1002/bmc.2679.

16. M.K. Matta, N.R. Pilli, J. Inamadugu and J.V.L.N.S. Rao, Acta Pharm. Sin. B, 2, 472 (2012); doi:10.1016/j.apsb.2012.07.003.

17. M.K. Matta, N.R. Pilli and J.V.L.N.S. Rao, Acta Chromatogr., 27, 27 (2013); doi:10.1556/AChrom.27.2015.1.3.

18. S. Putluru, M. Matta, D. Ahire, M. Subramanian, M. Sinz and S. Mandlekar, Drug Metab. Lett., 10, 264 (2017); doi:10.2174/1872312810666161124143522.

19. US DHHS, FDA, CDER, CVM. Guidance for Industry (Draft): Bioanalytical Method Validation. U.S. Department of Health and Human Services, Food and Drug Administration, Center for Drug Evaluation and Research (CDER), Center for Veterinary Medicine (CVM), 2013, Rockville, MD, USA. 